\title{
MIXED GEOSPATIAL METHODS BASELINE STUDY TO EVALUATE AND MODEL GENTRIFICATION ALONG THE WESTSIDE ATLANTA BELTLINE, USA
}

\author{
A. Aragón ${ }^{\mathrm{a}}$, M. C. Johnson Gaither ${ }^{\mathrm{b}}$, M. Madden ${ }^{\mathrm{a}}$
}

\author{
${ }^{a}$ Center for Geospatial Research (CGR), Department of Geography, University of Georgia, Athens, GA 30602, USA \\ (amanda.aragon, mmadden)@uga.edu \\ ${ }^{\mathrm{b}}$ United States Department of Agriculture (USDA) Forest Service, Southern Research Station, Athens, Georgia, 30602, USA \\ (cassandra.johnson@usda.gov)
}

Commission IV, WG IV/8

KEY WORDS: Agent Based Modeling, Sentinal-2 MSI, Atlanta, Survey, Greenspace, Southeastern United States

\begin{abstract}
:
There is a need for sustainable infrastructure planning that includes environmental, social, economic, and transportation management consideration. A portion of a former railway corridor around Atlanta, Georgia was converted to a multi-use trail called the Atlanta BeltLine to provide walking and bicycling access to businesses and homes. Although the pedestrian-transit trail brings renewed development and services, it also triggers increases in home values and displaces existing communities. This study uses remote sensing and geospatial analysis to focus on changes in the structural conditions of housing along the Westside segment of the Atlanta BeltLine. The first segment of the Beltline, the Eastside Trail completed in 2007, demonstrated an alarming influx of development. Increases in property values opened the doors for private investors to purchase properties and open new businesses in communities adjacent to the BeltLine. The proposed Westside segment of the BeltLine has raised concerns by community members who fear the impacts of gentrification. An assessment of housing conditions was conducted by researchers ranking the structural conditions of homes adjacent to the Westside BeltLine. To conduct an estimate of green spaces along both segments of BeltLines, Sentinel-2 Earth Observation Satellite was used to calculate the percentage of green spaces in. A preliminary concept agent-based simulation model (ABM) is being developed to forecast human movement and housing condition around the BeltLine. Combined, the housing survey, green space inventory, and ABM model provides a baseline method to record and identify social and the environmental impacts.
\end{abstract}

\section{INTRODUCTION}

With an alarming population increase of over 35\% and 75,000 per year within the last ten years, the Atlanta Metropolitan area is among one of the top ten in population growth in the U.S. (U.S. Census Bureau, 2019). The changing demands due to population growth and need for infrastructure necessitates the need for repurposing unused open greenspaces and greenways. The Atlanta BeltLine is an example of a greenway passage. The concept of the Atlanta BeltLine began in 1999 as a Master's Thesis project proposed by Ryan Gravel. At the time, Gravel was a Georgia Tech architecture and city planning student who aimed to design a walkway utilizing the abandoned historic rail corridor loop. The passageway runs $35 \mathrm{~km}$ around the outer perimeter of Atlanta and connects 45 inner-city communities. In 2001, the idea was proposed to the City Council (Burns, 2014). Gravel himself stated in a 2005 addendum to his 1999 Master's Thesis, "The grassroots support built so early for the Beltline rests not on abstract theory, but on the intuitive nature of the proposal," and describes how it has expanded beyond his original vision into values of communities (Gravel, 2005). There are currently two main sections of the BetlLine already constructed, the Eastside and the Westside (Figure 1).

With over a million visitors yearly on the Eastside Trail, the BeltLine has successfully met the original intention of the project. The installation of the Atlanta BeltLine provides walking and bicycling passage to thousands of businesses and homes. There are estimates of up to 1 billion U.S. dollars from private investors that have contributed to the growing economic boom of neighbourhoods along the BeltLine However, there is concern about what the effect of such a large-scale installation will have on intensifying gentrification, generally defined as the changes in the character of a neighbourhood by attracting more affluent residents and businesses. In 2017, the Westside Trail was completed and it is expected to generate similar effects of increased home restoration, rent rates and influx of commercial services in the traditionally African American community of the Eastside.

\section{STUDY PURPOSE}

2.1 This study is developing an integrative methodology that combines street-view to satellite remote sensing observations and geospatial analysis to asses gentrification of the neighbourhoods surrounding the newly built Eastside Trail of the BeltLine, as compared to the perviously constructed Westside Trail. We applied multiple methods to look at gentrification, including a ground suveywhere we video recorded and visually inspected the front street-views of homes at three points in time and assessed changes in home condition. The second method classified medium-resolution satellite imagery to characerize the relative amounts of natural vegetation and urban development in proximity of the BeltLine segments. The third method incorporates a conceptualization of gentrification driven by the introduction of green infrastructure in an urban space and develops an Agent-Based Model (ABM) that simulates changes in houing conditions withgentrification related to greenways.

2.2 We selected the Westside segments of the BeltLine to examine gentrification by assessing changes in the condtions of dwelings within a block buffer of the BeltLine (i.e., aproxomently two streets on either side of the BeltLine). The oppurtunity to conduct a longitudinal study serves both as a benchmark and provides a preliminary methodology for change analysis. 


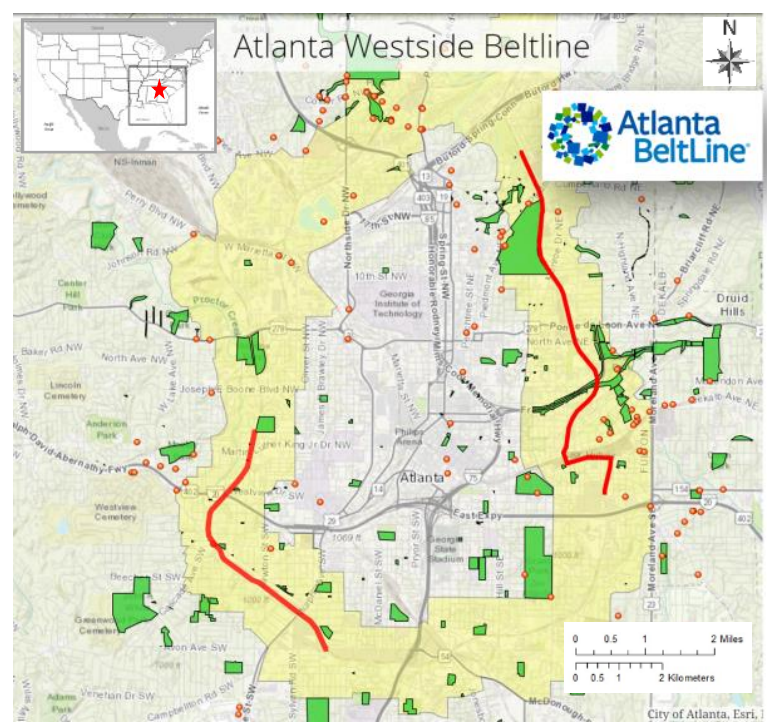

Figure 1. The locations of both the Eastide and Westside segments of the Atlanta BeltLine are shown in red. The yellow area is the neighbourhood with in the $35-\mathrm{km}$ proposed corridor. Green represents parks.

\section{METHODOLOY}

\subsection{Sentinel-2 Data Acquisition}

Satellite data were acquired to look at the vegetation and builtup urban context of neighbourhoods along both segments of the BeltLine. Typically, in U.S. urban areas with higher minority populations there is less greenspace and the Westside segment is in an area with a higher minority population. Georectified cloud-free Sentinel-2 MultiSpectral Instrument (MSI) images from Summer 2017 were downloaded from the United States Geological Survey (USGS) using EarthExplorer (earthexplorer.usgf.gov). A mid-summer image was selected to express the maximum levels of greenness during the growing season. Sentinel-2 multispectral scenes at a $10-\mathrm{m}$ resolution were acquired to conduct a supervised classification of greenspaces, parks and trees, commercial buildings, houses and roads of the Atlanta-Metro area. A $2.5-\mathrm{km}$ buffer of the current segments of the BeltLine was applied to create our area of interest (Figure 2). This analysis was done to help understand opportunities residents in these neighbourhoods have for greenspace interactions apart from the BeltLine.

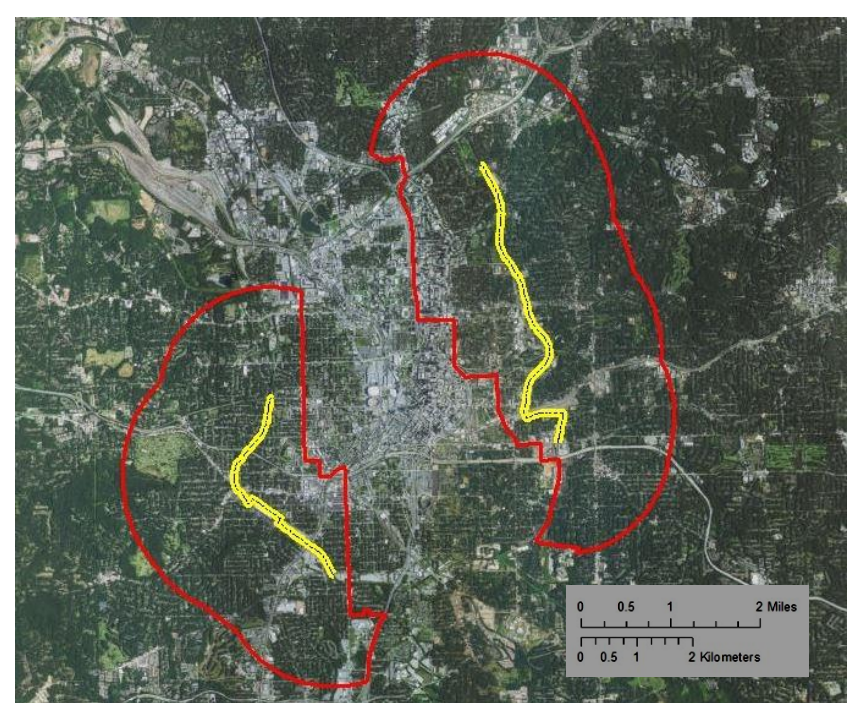

Figure 2. Sentinal-2 MSI colour composites showing the area affected within a $2.5-\mathrm{km}$ buffer of both East and West segments of the Atlanta BeltLine: acquired on May 5, 2017.

\begin{tabular}{|c|c|c|}
\hline $\begin{array}{l}\text { Scene ID/ } \\
\text { Identifier }\end{array}$ & $\begin{array}{c}\text { Date of } \\
\text { Acquisition }\end{array}$ & $\begin{array}{c}\text { Satellite / } \\
\text { Sensor }\end{array}$ \\
\hline $\begin{array}{l}\text { S2A_MSIL1C_201705 } \\
\text { 15T162341_N0205_R0 } \\
\text { 40_T16SGC_20170515 } \\
\text { T163103 }\end{array}$ & $\begin{array}{l}2017-05-15 \\
\text { T:16:23:41.026Z }\end{array}$ & Sentinel-2 / MSI \\
\hline $\begin{array}{l}\text { S2A_MSIL1C_201705 } \\
\text { 02T161351_N0205_R1 } \\
\text { 40_T16SGC_20170502 } \\
\text { T162229 }\end{array}$ & $\begin{array}{l}\text { 2017-05-02 } \\
\text { T16:13:51.026Z }\end{array}$ & Sentinel-2 / MSI \\
\hline
\end{tabular}

Table 1. Sentinal-2 images used in this study

\subsection{Satellite Classification and Inventory}

A satellite remote sensing inventory was adapted from Barlow's "Mapping Urban Green Infrastructure" to develop and generate a percent value of green areas in and surrounding metro Atlanta (Barlow, 2018). The method allows researchers to evaluate social-economic concerns. The advantage of using satellite imagery is the broad-area classification of the environmental conditions characterizing the greenness of neighbourhoods. It delineates values from a broad-scale area of interest to evaluate the relationships that exist between the natural environment and the physical, socio-economic, health and landscape characteristics.

An unsupervised classification was performed to identify general land use/land cover classes and Normalized Difference Vegetation Index (NDVI) computed to map vegetation cover within the $2.5-\mathrm{km}$ buffer zone with the metro areas removed (Figures 3 and 4; Equation 1). The vegetation layer was used to identify the main greenspaces for comparison between the Eastside and the Westside neighbourhoods.

$$
N D V I=\frac{N I R-R E D}{(N I R+R E D)}(1)
$$

where $\quad N I R=$ near infrared spectral band $R E D=$ red spectral band 


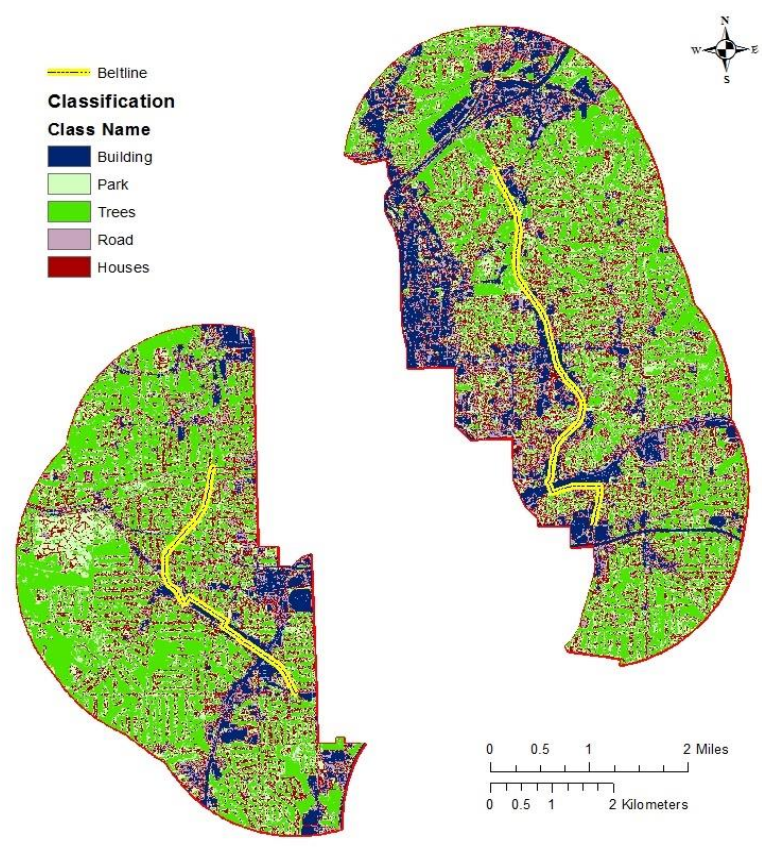

Figure 3. Unsupervised classification of the Westside and Eastside BeltLine at a $2.5-\mathrm{km}$ buffer with metropolitain neighbourhoods removed from the center.

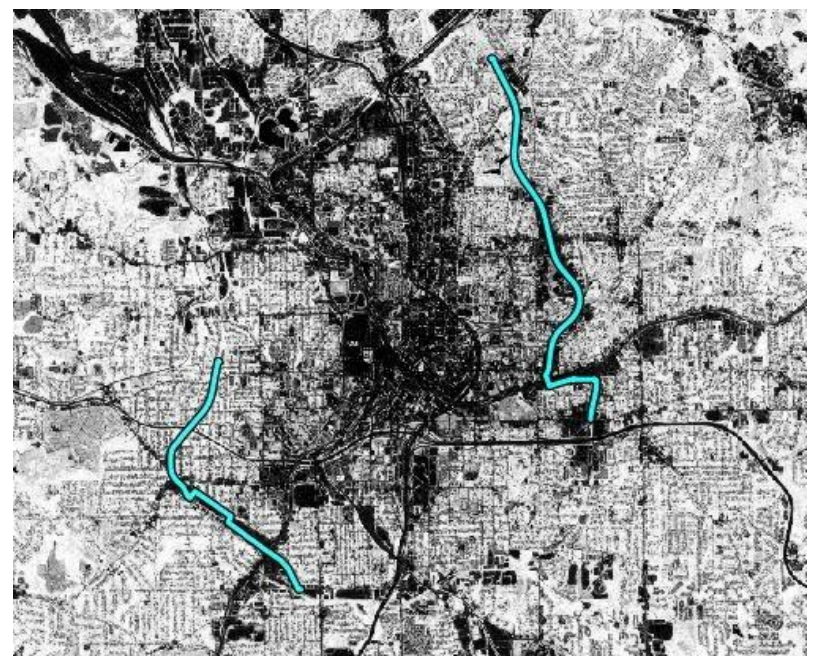

Figure 4. Greyscale NDVI of Metro Atlanta from midsummer 2017 Sentinel-2 scene. Eastside and Westside Beltline shown in light blue.

\subsection{Ground Survey of Houses}

To assess gentrification at the street level, we conducted a housing survey that evaluated the front of the homes adjacent to the BeltLine. The survey was adapted from the rating scale found in "Developing a Framework for Neighbourhood Housing Condition Evaluation" prepared by students in the University of South Florida Urban and Regional Planning program (Yassin and Mahler, 2011). This tool is used to asses housing conditions and create an inventory of the building stock based on four criteria: roofing, siding/stucco, windows, and landscape. In this research the visual observations used to inventory the condition of the homes were given a rating of 0 , 10,15 , and 25 to indicate perfect conditions, slightly damaged, significant damage, and completely dilapidated attributes, respectively. The process for the BeltLine survey was streamlined by assigning a Dilapidation Index combining all four values to equal between 0 to 100 (Figure 5).

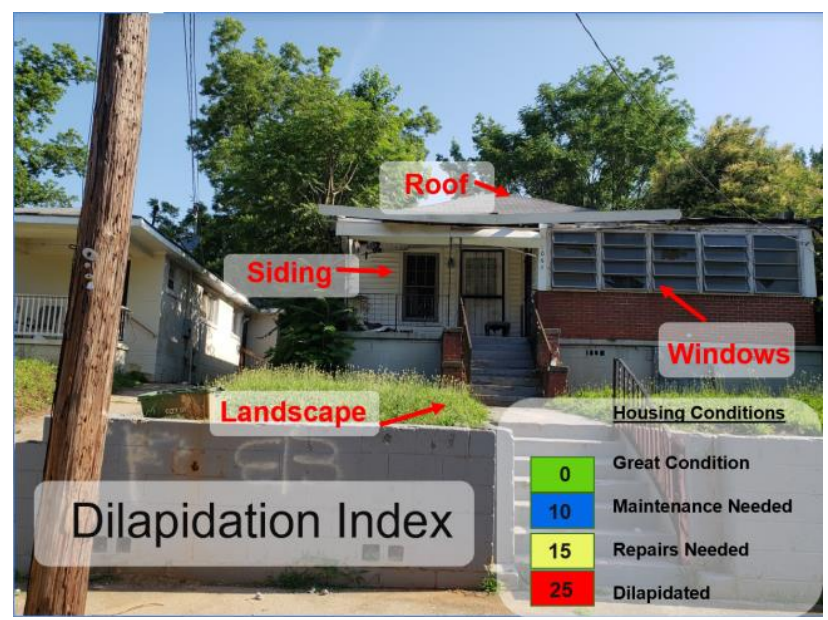

Figure 5. Example of a home along the BeltLine and the Dilapidation Index ranking system used when visually inspecting the condition of the front of the home.

\subsection{Ground Survey Preparation}

To prepare for the ground survey, parcel maps were used to identify residential housing units that are within one block or adjacent to the Beltline. Once the units were identified, Google Maps was used to create an automated GPS map to efficiently navigate the streets in order to conduct the street-view survey. An Uber driver was instructed to operate the vehicle while observations were made for each house by researchers of this study. The driver followed the Google Map driving instructions while the researchers systematically evaluated the house fronts and assigned values of $0-25$ based on the four observed conditions of the homes from the street view.

3.4.1 2018 Ground Survey: In 2018, a baseline housing survey was conducted by utilizing the Uber driver to systematically tour areas around the BeltLine as researchers visually recorded and quantified the structural conditions of homes adjacent to the Westside BeltLine. To prepare for the survey, parcel maps from Computer Assisted Mass Appraisal (CAMA) data sets for Fulton County were used to identify residential housing units that are within one block or adjacent to the Beltline (Pippin et al., 2017). The survey started in Fall 2018 and required five re-visits over the course of two months. The roads were identified ahead of time using Google EarthPro to enter a path and provide direction to the driver.

3.4.2 2019 Ground Survey: In 2019, the survey was repeated to evaluate the quality of the same homes using the Dilapidation Index ranking system. This time, a camcorder was used to capture the conditions of the homes. The videos were later reviewed and evaluated in-house to assign the values and calculate the Dilapidation Index. Researchers decided to use the camcorder method for the revisit because the survey could be conducted in one day rather than over the span of months and it also documented housing conditions for particular points in time. 


\subsection{Agent Based Model (ABM)}

Based on the street-view ground surveys, changes in Dilapidation Indices and satellite-based greenness characterization of neighbourhoods, an ABM conceptual model was created. The ABM focuses on the structural conditions of housing and area of greenspaces to demonstrate social constraints along the newly completed Westside segment of the Atlanta BeltLine, surrounding environments, and historical sites. Based on known and well-tested models, a model was built from revised code to add additional information that mimics and synthesizes actual events as observed along the Atlanta BeltLine (Railsback, 2019).

3.5.1 Agent Based Model Preliminary Planning: The first stage of developing this ABM is representing concepts of gentrification drivers in an urban setting with changing greenspace (Figure 6). The following stage will incorporate the proportions of homes along the Westside segment of the BeltLine itself and the concept model will forecast future socioeconomic developments and ecological changes in greenspace.

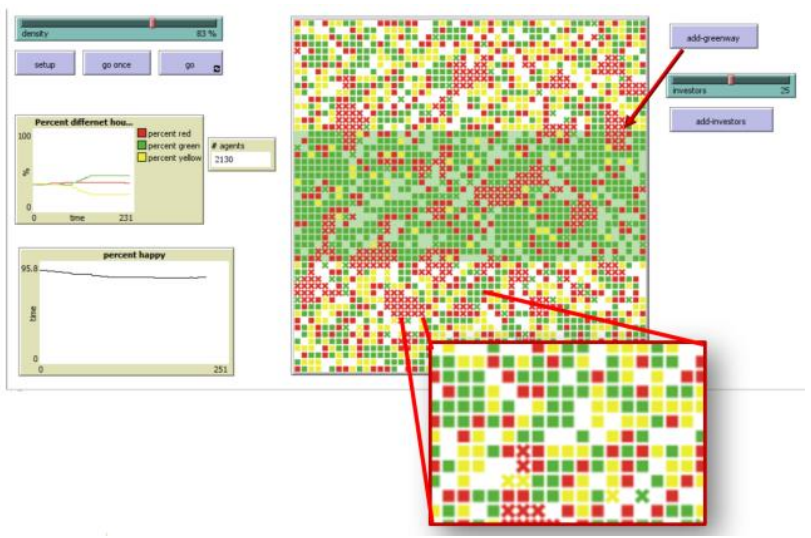

Figure 6. Screen capture of the conceptual ABM depicting housing condition changes as the BeltLine (green bar) is introduced. Each coloured square within the grid represents one home randomly located in proximity to the greenway.

3.5.2 Happiness Scale - Agent Based Model Preliminary Planning: Figure 6 models the behaviour of three types of gentrification agents in a neighbourhood. The red agents and yellow agents represent somewhat dilapidated homes, while green agents are homes that are in good to excellent condition. The red and yellow agents get along well with one another. In addition, each agent wants to make sure that it lives near some of "its own kind." That is, each red agent wants to live near at least some red agents, and each green agent wants to live near at least some green agents. The simulation shows how these individual preferences ripple through the neighbourhood as the greenway area increases and gentrification processes occur, leading to interesting broad-scale patterns in time and space.
3.5.3 Adding a Greenway: When a greenway is added, it increases the value of the homes and the conditions become more favourable for the highly ranked green agents. The number of new investors can also be adjusted to account for the number of people moving into the properties. Another element of this simulation is the "Happiness" of the agents as indicated by the red, yellow and green agent color-coded symbol ' $x$ '. This project was inspired by Thomas Schelling's writings about social systems such as housing patterns in cities as described by Wilensky (1997).

\section{RESULTS AND DISCUSSION}

\subsection{Preliminary Housing Observations}

One block along the BeltLine was reviewed to demonstrate a sublocation of the changes that occurred from one year to another, 2018 to 2019. Upon re-survey in 2019, it was observed that in this particular neighbourhood, $60 \%$ of the homes had undergone some form of restoration and/or improved changes from dilapidated to either good or livable condition within the year (Figure 7).
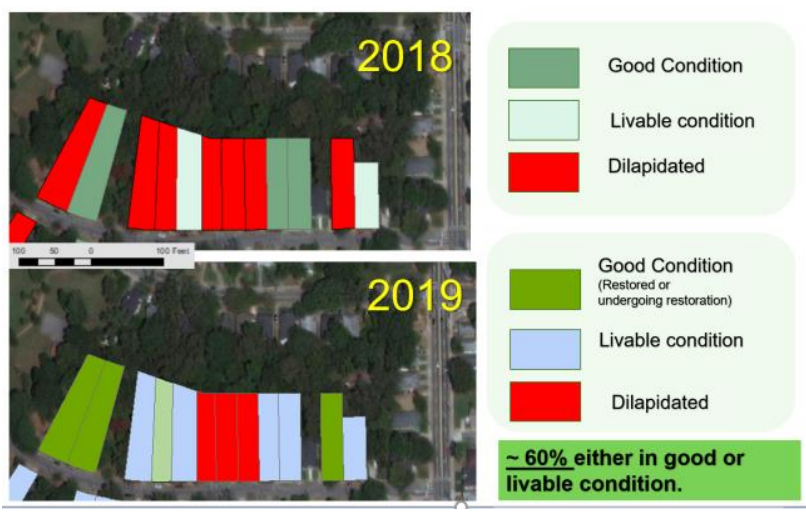

Figure 7. Sample of the changes from 2018 to 2019 on one block of the ground-surveyed area. From the street-view observations and video recordings, there appeared to be a $60 \%$ improvement in housing conditions within one year.

\subsection{Sentinal-2 Greenness and Urban Area Results}

This project serves as an initial greenness, commercial buildings and residential housing characterization and framework for future surveys along the Atlanta BeltLine. Although this is a preliminary introduction to the proposed study, the research conducted provides a clear and systematic approach to identify, quantify, and map existing conditions of houses and green spaces in communities that are in close proximity to the BeltLine. The satellite approach characterizes overall greenness of the area by calculating NDVI values collected from a $10-\mathrm{m}$ resolution scene from midsummer 2017. 


\begin{tabular}{|l|l|c|c|c|c|c|}
\cline { 2 - 7 } \multicolumn{1}{c|}{} & \multicolumn{3}{c|}{$\begin{array}{c}\text { Westside Trail \% } \\
\text { Classifications }\end{array}$} & \multicolumn{3}{c|}{$\begin{array}{c}\text { Eastside Trail \% } \\
\text { Classifications }\end{array}$} \\
\hline Class & $\begin{array}{l}\text { Sq } \\
\text { meters }\end{array}$ & Hectares & \% & $\begin{array}{l}\text { Sq } \\
\text { meters }\end{array}$ & Hectares & $\%$ \\
\hline Building & 238,200 & 23.820 ha & $8 \%$ & 597,660 & 59.799 ha & $15 \%$ \\
Parks & 672,960 & 67.296 ha & $\mathbf{2 3 \%}$ & 890,970 & 89.087 ha & $\mathbf{2 3 \%}$ \\
Trees & $1,240,920$ & 124.092 ha & $\mathbf{4 2 \%}$ & $1,282,870$ & 128.287 ha & $\mathbf{3 2 \%}$ \\
Roads & 260,130 & 26.013 ha & $9 \%$ & 505,460 & 50.546 ha & $13 \%$ \\
Houses & 529,550 & 52.955 ha & $18 \%$ & 782,550 & 78.255 ha & $19 \%$ \\
Total & $\mathbf{2 , 9 4 1 , 7 6 0}$ & $\mathbf{2 9 4 . 1 7 6 ~ h a ~}$ & & $\mathbf{4 , 0 5 9 , 5 1 0}$ & $\mathbf{4 0 5 . 9 5 1 ~ h a ~}$ & \\
& & & & & & \\
\hline
\end{tabular}

\begin{tabular}{|l|l|}
\hline Total \% Greenspaces & $\begin{array}{l}\text { Westside 65\% } \\
\text { Eastside 55\% }\end{array}$ \\
\hline
\end{tabular}

Table 2 Total \% Greenspaces in BeltLine neighbourhoods and 2.5- $\mathrm{km}$ buffer from both Eastside and Westside BeltLine segments.

\subsection{Agent Based Model Preliminary Planning}

The ABM model is highly adaptable and can also be used to synthesize and analyse other greenways located within the U.S., and with adjustments to the rules of gentrification agents, also in other countries. As demonstrated, there are interactions among the agents that reflect the ability to make decisions and apply to a "Happiness" or sentimental scale. Further development of the model will depend upon feedback from project partners and ongoing longitudinal research. Overall the final model appeared to successfully represent gentrification agents acting in the Atlanta, Georgia, USA area. Although no actual numerical values are available in the conceptual ABM, the model represents the emerging characteristics of the observed behaviour along the Atlanta BeltLine. ABM provides a new perspective on an ongoing concern of both the observed conditions and the communities' desires to maintain the integrity of their neighbourhoods. City planners can consider emerging behaviours and plan accordingly to accommodate both the investors and the community members located in these delicate zones.

\section{CONCLUSIONS}

\subsection{General Conclusions}

The incorporation of mixed geospatial methods to understand the interactions of environmental and social behaviours related to greenway infrastructure and gentrification is a growing field in research and crosses many disciplines. Street-view ground surveys allow researchers to assess and rank the quality of housing conditions using a new Dilapidation Index and video documentation of house fronts. Satellite imagery provides a broad-view of overall land use/land cover characterizing the natural vegetation and built-up urban environment. Conceptual ABMs allow researchers to create a simple demonstration to explain complex systems. This model particularly embraces the opportunity to accommodate an ongoing debate of gentrification considering a delicate balance among agents of improved urban environments while maintain community identities and integrity of traditional neighbourhoods in a developing urban setting.

\subsection{Future Work}

Future work will develop a hedonic model that will evaluate the intervention of the greenway as a social impactor on the housing market. This statistical approach will consider the spatial patterns of ecological systems and how they affect the agents' choices and governing rules (Brasington, 2014). This will provide a more robust statistic assessment and will contribute to the ability to derived real-world values within the ABM.

\section{ACKNOWLEDGEMENTS}

The authors wish to acknowledge support of the USDA Forest Service Southern Research Centre; University of Georgia, Department of Geography, Centre for Geospatial Research; and our Uber driver.

\section{REFERENCES}

Burns, Rebecca (May 6, 2014) "Can Atlanta Go All In on the BeltLine" CityLab, "The Future of Transportation: How getting from her to there is changing forever" retreived 4-4-2018 https://www.citylab.com/life/2014/05/can-atlanta-go-allbeltline/9036/

Brasington, D. M. (2014). Housing choice, residential mobility, and hedonic approaches. Handbook of Regional Science. Springer-Verlag, Berlin: Heidelberg, Germany, 147-165.

Gravel, R. A. (2005). "Addendum to the Beltline Thesis" Belt line-Atlanta: design of infrastructure as a reflection of public policy (Doctoral dissertation, Georgia Institute of Technology, 1999).

https://smartech.gatech.edu/bitstream/handle/1853/7400/Beltlin e_2005addendum.pdf

Pippin, S., Jones, S., \& Johnson Gaither, C. (2017). Identifying potential heirs properties in the southeastern United States: A new GIS methodology utilizing mass appraisal data (Technical Report SRS-225). Southern Research Station, USDA Forest Service. srs.fs. usda.gov/pubs/gtr/gtr_srs225.pdf

Railsback, S. F., \& Grimm, V. (2019). Agent-based and individual-based modeling: a practical introduction. Princeton university press.

Wilensky, U. (1997). NetLogo Segregation model. http://ccl.northwestern.edu/netlogo/models/Segregation. Center for Connected Learning and Computer-Based Modeling, Northwestern University, Evanston, IL.

U.S. Census Bureau. (2019, April 18) New Census Bureau Estimates Show Counties in South and West Lead Nation in Population Growth, Retrieved from https://www.census.gov/newsroom/pressreleases/2019/estimates-county-metro.html

Yassin, Aiah., Mahler, Kenneth Scott., Storm, Elizabeth. (2011). Developing a Framework for Neighbourhood Housing Conditions Evaluations: The Neighbourhood of Lealman. Urban Contemporary Issues, University of South Florida, 33 p. 